\title{
Modelo interaccional comportamental de tránsito: algoritmo de diagnóstico
}

\author{
Interactional behaviourist transit model: diagnosic algorithm
}

\author{
Luciano Petit \\ Universidad Abierta Interamericana \\ lucianopetit@gmail.com
}

Fecha de recepción: 14 de marzo de 2016 / Fecha de aprobación: 14 de junio de 2016

\section{RESUMEN}

El presente artículo tiene como finalidad presentar una nueva perspectiva teórica y técnica articuladora de saberes, como así también de campos disciplinares disímiles pero necesarios y complementarios para analizar y comprender la complejidad que demandan todas las situaciones, eventos y fenómenos referidos al tránsito y seguridad vial. Se exponen, por un lado, los principios conceptuales del Trans- Sistema Tránsito y Seguridad Vial y del Modelo Interaccional Comportamental de Tránsito y, por el otro, el desarrollo técnico para la obtención de un punto sintetizador, o puntos sintetizadores, que permite calificar dicho sistema a partir de cuatro condiciones. El valor se obtiene mediante un algoritmo que propone la intersección de rectas en un plano de ejes cartesianos. El mismo se ejecutó con el software $\mathrm{MATLAB}^{\circ}$, resultando de la acción del software gráficos que posibilitan diagnosticar (y diagnóstico en sendero) el Trans- Sistema Tránsito y Seguridad Vial, a través del Modelo Interaccional Comportamental de Tránsito, vinculando elementos físicos, por ejemplo infraestructura vial, y comportamientos, por ejemplo conductas de automovilistas urbanos.

La teoría y la técnica son la expresión de una frágil interacción entre actores (factor psicosocial) y elementos físicos (entorno físico) a partir de la estructura irreductible de sus componentes, manteniendo la distinción entre actores y elementos físicos en asociación dialéctica de interdependencia e integridad que incluye extremos disímiles: Sistema Comportamental y Sistema de Elementos Físicos.

PALABRAS CLAVE: sistema; tránsito; comportamiento; físico.

\section{ABSTRACT}

The aim of this article is to present a new theoretical and technical perspective which coordinates knowledge as well as disciplinary, though dissimilar, fields that are necessary and complementary for the analysis and understanding of the complexity of all situations and events relating to transit and road safety. On the one hand, the conceptual principles of Trans-System Transit and Road Safety and the Interactional Behavioural Transit Model are set out; on the other hand, there follows the technical development, in order to obtain a synthesizing point, or points, which will allow us to define such system based on four conditions. The value is obtained using an algorithm that proposes the intersection of lines on a plane of Cartesian axes. It has been run with MATLAB TM software, resulting in graphics which make it possible to diagnose ("path diagnosis") the TransSystem Transit and Road Safety, through the Interactional Behavioural Transit Model, linking physical elements such as road infrastructure, and behaviours such as behaviour in urban drivers.

The theory and the technique result from a fragile interaction between actors (psychosocial environment) and physical elements ( physical environment), based on the irreducible structure of their components, and keeping the distinction between such actors and physical elements in a dialectical association of interdependence and integrity that includes dissimilar extremes: Behavioural System and System of Physical Elements.

KEYWORDS: system, transit, behaviour, physical. 


\section{INTRODUCCIÓN}

El objetivo del presente artículo es describir el proceso constructivo de una nueva perspectiva teórica y técnica nacida como respuesta a la pregunta sobre la posibilidad de relacionar dialécticamente comportamientos y elementos físicos en el tránsito y seguridad vial. Y consecuentemente, asociar saberes de campos disciplinares disímiles pero necesarios y complementarios para analizar y comprender la complejidad que demandan todas las situaciones, eventos y fenómenos referidos al tránsito y seguridad vial. El desarrollo conceptual e instrumental que se expondrá fue la primera etapa del plan de investigación que actualmente se encuentra finalizando la última etapa. Principalmente el diseño y desarrollo técnico: la obtención de un punto sintetizador, o puntos sintetizadores, que permite calificar dicho sistema a partir de cuatro condiciones; este valor se obtiene mediante un algoritmo que propone la intersección de rectas en un plano de ejes cartesianos. Los algoritmos se ejecutaron con el software MATLAB ${ }^{\circledR}$ (versión R2014a del 2014), resultando de la acción del software gráficos que posibilitan dar cuenta del diagnóstico, magnitud y predicción del Trans- Sistema Tránsito y Seguridad Vial a través del Modelo Interaccional Comportamental de Tránsito. La labor de desarrollo algorítmico en el software $\mathrm{MATLAB}^{\circ}$ se dividió en tres instancias de diseños de códigos de ejecución de diversas funciones: la primera, posibilita la realización del diagnóstico, y diagnóstico en sendero; la segunda, conlleva la composición de la magnitud del diagnóstico; y la última, permite efectuar un diagnóstico predictivo. De la primera de las tres instancias se detallará la ecuación resultante y expondrá el diseño del algoritmo desarrollo con el software MATLAB ${ }^{\bullet}$, más su ejecución.

\section{SISTEMA TRÁNSITO Y SEGURIDAD VIAL}

Posicionado el factor humano como la causa primordial de los siniestros viales, se plantea el inconveniente de que dicho concepto culmina transformándose en un "cajón de sastre" (Lopez-Araujo \& Osca Segovia, 2007; Izquierdo \& Torres, 2009); metáfora que ilustra que todo es factor humano, imposibilitando diferenciar cuales son actores involucrados y las relaciones entre ellos, y sus comportamientos (Petit, 2014). La psicología del tránsito se ha ocupado de la seguridad vial focalizándose en el estudio del factor humano como primordial causante de riesgos, y su incidencia (Rothengatter, 1997).

Los estudios en materia de seguridad vial se han concentrado en la conducción (Huguenin \& Rumar, 2001) y las actitudes de riesgo (Iversen, 2004), de manera que los principales modelos teóricos en psicología del tránsito se dirigieron en esta dirección: modelos motivacionales y de riesgo (Näätänen \& Summala, 1974;
Wilde, 1982); modelos jerárquicos de la tarea de conducción (Michon, 1985); y los modelos de error humano (Reason, 1990). Cuya derivación ha sido la menor atención prestada al estudio de otros usuarios: motociclistas, ciclistas y peatones, como así también otros elementos: la accesibilidad o sustentabilidad del sistema de tránsito, y no únicamente la seguridad vial (Ledesma, Poó \& Montes, 2011). El factor humano aporta componentes válidos para aprehender sistémicamente el problema, siendo un elemento más sin omitir al factor social: la interacción social y los valores sociales (Izquierdo, Torres \& Martínez, 2013).

La búsqueda de distinguir, clarificar, ordenar, clasificar y puntualizar el "cajón de sastre" es el origen del Sistema Tránsito y Seguridad Vial [STSV] (ver figura 1) planteamiento y enfoque postulado y desarrollado por Petit (2015), que propone estructurar, regularizar y organizar las múltiples interacciones de los principales actores que lo influyen y determinan, limitan y describen, delineando un sistema dinámico de integración y diferenciación; es decir, configurando el factor psicosocial como estructura dialéctica.

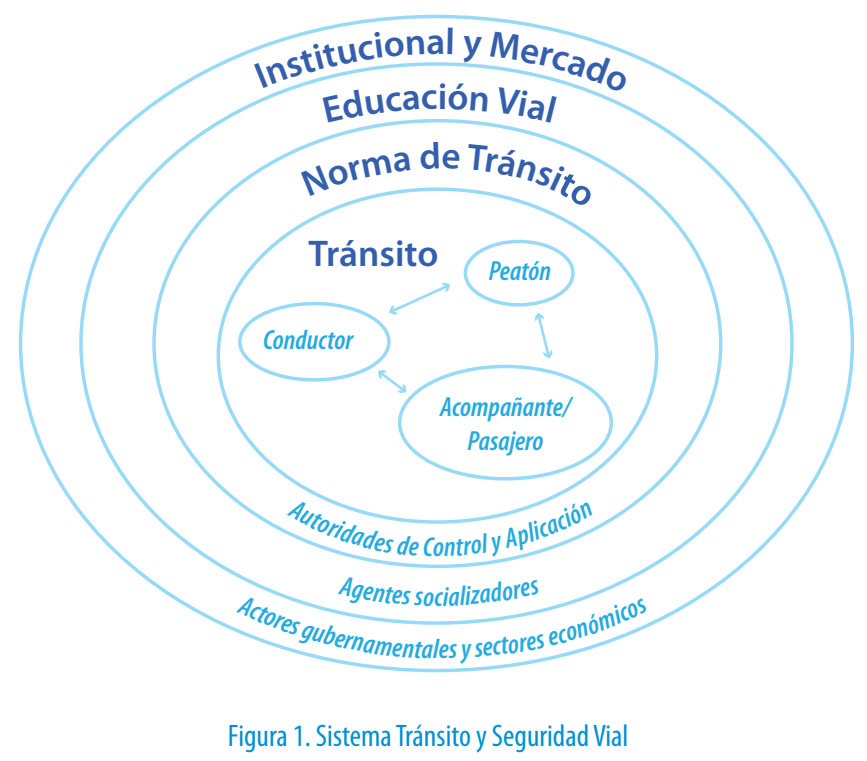

Para ello, se tienen en cuenta cuatro subsistemas que lo componen, integran y constituyen: Tránsito, Norma de Tránsito, Educación Vial e Institucional y Mercado. Distinguiéndose por los actores, sujetos e instituciones que los componen $\mathrm{y}$, fundamentalmente, por los niveles de interacciones. Es decir, interviene un proceso de coordinación e integración de las interacciones correspondientes; expresando el STSV un pasaje inicial de indiferenciación de actores e interacciones a diferenciaciones de las mismas, y posteriormente, su integración, intra-subsistemas e inter-subsistemas. Las figuras y vínculos de los cuatro subsistemas, donde los niveles previos se subsumen 
e integran al nuevo subsistema que es habilitado por ellos y se cimienta sobre el soporte efectivo de las asociaciones intra e inter subsistemas que se realicen, son:

1. Subsistema Tránsito: el ámbito de observación se centra en los usuarios de la vía pública, entendidos como espacio que ocupan y excluyendo su carácter o condición de ente. Los sujetos destacados aquí son: conductor, acompañante y/o pasajero y peatón.

2. Subsistema Norma de Tránsito: su órbita de injerencia se limita al código de tránsito que regula la circulación en la vía pública; es decir, a las autoridades que velan por su control (cumplimiento) y aplicación. Los sujetos relevantes son: policía y/o agentes de control de tránsito y autoridades judiciales.

3. Subsistema Educación Vial: el agente socializador abarca a toda institución y persona que ejerza la práctica educativa, y se diferencian por su pertenencia dentro del universo educativo: educación formal, educación no formal y educación informal. El grupo de educadores se integra entonces por padres, pareja, amigos, instructores, maestros y profesores, medios de comunicación, Tecnologías de la Información y Comunicación y asociaciones sin fines de lucro, entre otros.

4. Subsistema Institucional y Mercado: incluye, por un lado, el sector gubernamental de toma de decisiones en materia de tránsito y seguridad vial. Los sujetos preponderantes son los actores políticos que integran el poder legislativo y el ejecutivo. Y por otro, el sector económico, público y privado, involucrado directamente e indirectamente en el área automovilística, transporte y vialidad. Los actores preponderantes son las empresas e industrias del sector.

En síntesis, el proceso constructivo de interdependencia de los subsistemas involucrados da lugar a un sistema que avanza del subsistema anterior al posterior, reorganizándose en la construcción; pasaje de las propiedades internas de cada subsistema al establecimiento de vinculaciones entre ellos, y ulteriormente, a la creación de relaciones sistémicas entre las asociaciones de los subsistemas; es decir, constituyéndose relaciones intra-Subsiste e inter-Subsistema.

\section{MODELO INTERACCIONAL COMPORTAMENTAL DE TRÁNSITO}

Los modelos predictivos de siniestralidad vial poseen una destacada importancia y variados usos para el análisis actual de la seguridad pero la calibración de estos modelos no es sencilla (Persaud, Lord \& Palmisano, 2002). El Modelo Interaccional
Comportamental de Tránsito [MICT] (Petit, 2015) se diferencia de ellos a partir de sus objetivos: en primer lugar, determinar las situaciones y condiciones en las que se encuentra el STSV, sistema que permite conceptualizar el factor psicosocial, y su construcción a partir de los actores y sus interacciones, de cada subsistema (Tránsito, Norma de Tránsito, Educación Vial e Institucional y Mercado), como de la vinculación entre ellos; y en segundo lugar, integrar y fusionar los comportamientos de los actores, de los diferentes subsistemas, con los elementos físicos y materiales del entorno propios de cada uno de los subsistema, los cuales enmarcan los comportamientos de los actores. Es decir, poder establecer los parámetros de su dinámica para afrontar la diversidad, variedad y variabilidad de situaciones, eventos, circunstancias e identidades que se desarrollan en el STSV.

El modelo es integrado por comportamientos distribuidos en los ejes Y (Y1 e Y2), por un lado, y elementos y/o comportamientos distribuidos en el eje X (X1 y X2), por el otro. El eje Y se integra por un solo tipo de comportamiento (conductas, pensamientos y emociones de iguales actores en las mismas condiciones y situaciones, delimitación determinada por el Subsistema), siendo Y1 el Comportamiento YO que implica conductas, pensamientos y emociones propias, autopercibidas u observadas directamente, e Y2 el Comportamiento OTRO, en el cual intervienen conductas, pensamientos y emociones ajenas, percibidas $u$ observadas indirectamente. El eje X se compone por elementos y/o comportamientos (uno u otro o la combinación de ambos), diversos actores, elementos, situaciones y condiciones (igual o diferentes Subsistemas de un mismo o distinto Sistema). Este eje puede también estar integrado por el mismo comportamiento que el del eje Y, pero variando el actor, situación y/o condición; el eje X se distribuye en la Variable A y la Variable B.

El punto de intersección entre los ejes $\mathrm{Y}$ (valores $\mathrm{Y} 1$ e Y2) y X (valores X1 y X2), determina la situación y condición del MICT, en el que se desenvuelven los comportamientos y los elementos y/o comportamientos a partir de su ubicación en el cuadrante del plano, delimitado por las coordenadas positivas ([+]:0) y negativas ([-]: 6); indicando las condiciones en las que se encuentra el STSV:

A. Sistema en Armonía: punto de intersección ubicado en las coordenadas de los cuadrantes superiores izquierdo del plano.

B. Sistema en Disputa: punto de intersección ubicado en las coordenadas de los cuadrantes medios del plano.

C. Sistema en Disonancia: punto de intersección ubicado en las coordenadas de los cuadrantes inferiores derecho del plano.

D. Zona de Exclusión: punto de intersección ubicado en las coordenadas de los cuadrantes inferior izquierdo o superior derecho del plano. 
Las condiciones varían en su movimiento (estabilidad), solvencia (control), y proyección (se integra por la combinación de flexibilidad y rigidez, capacidades aportada la primera por el movimiento y la segunda resultante de la solvencia). A excepción de la Zona de Exclusión en la que no es factible, sistémicamente, la interacción, asociación y vinculación entre los actores y elementos y/o actores, la contradicción de los mismos como antagónicos da lugar a la ausencia de articulación; es decir, conlleva una oposición real entre dos realidades antagónicas, expresándose una negación concreta al darse una acción y su negación real en donde puede ser: ambas verdaderas, ambas falsas o una verdadera y otra falsa.

El MICT presenta tres áreas de interacción, más la Zona de Exclusión (ausencia de vinculación), asignadas a los actores y elementos y/o actores:

1. Sistema en Armonía: las asociaciones armónicas de los actores y elementos y/o actores posibilitan un STSV Vial estable (invariabilidad de movimiento), solvente y con proyección de rigidez pero con capacidad de flexibilidad.

2. Sistema en Disputa: las interacciones en disputa de los actores y elementos y/o actores conllevan un STSV con inestabilidad (constante movimiento), insolvencia en el control de los comportamientos y/o elementos y/o actores, y con proyección de flexibilidad pero con capacidad de rigidez.

3. Sistema en Disonancia: las vinculaciones en disonancia de los actores y elementos y/o actores produce un STSV con anarquía de movimiento, insolvencia (incontrolable); sin rigidez y sin capacidad de flexibilizarse, como proyección.

4. Zona de Exclusión: los actores y/o elementos se repelen al presentar valores positivos y negativos extremos en uno y otro eje, por lo cual uno de ellos es expulsado del STSV.

El nivel, situación y capacidad de las condiciones de articulación dialéctica entre su movimiento, solvencia y proyección, se indica en la distancia de las polaridades, positivas ([+]: 0) y negativas ([-]: 6). La diferencia e indiferenciación de polaridad entre los comportamientos y elementos y/o comportamientos indica la situación y capacidad de movimiento, solvencia y proyección: a mayor distancia mayor deficiencia en las condiciones de interacción. No así en la Zona de Exclusión donde el eje con menor valor (positivo [+]) es expulsado del STSV.

En síntesis, el MICT (ver figura 2) se integra de cuatro componentes: Ejes: Y (Y1 e Y2) y X (X1 y X2).

Comportamientos y elementos y/o comportamientos: igual comportamiento (Comportamiento YO y Comportamiento OTRO) y elemento físico y/o comportamiento, uno u otro o la combinación de ambos, (Variable A y Variable B). Polaridades: (+) Positiva: 0; y (-) Negativa: 6. Situaciones de Interacciones Comportamentales de Tránsito: 1. Sistema en Armonía: zona superior izquierda, color verde; 2 . Sistema en Disputa: zona central, color amarillo; 3. Sistema en Disonancia: zona inferior derecha, color rojo; 4. Zona de Exclusión: zona inferior izquierda o margen superior derecho, color gris.

\begin{tabular}{|c|c|c|c|c|c|c|c|c|}
\hline \multirow{8}{*}{ 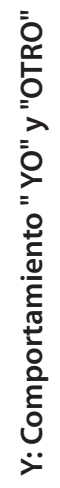 } & $(+)$ & 1 & 2 & 3 & 4 & 5 & 6 & $\begin{array}{l}(-) \\
(+)\end{array}$ \\
\hline & 1 & & & & & & & 1 \\
\hline & 2 & & & & & & & 2 \\
\hline & 3 & & & & & & & 3 \\
\hline & 4 & & & & & & & 4 \\
\hline & 5 & & & & & & & 5 \\
\hline & 6 & & & & & & & 6 \\
\hline & $\begin{array}{l}(-) \\
(+)\end{array}$ & 1 & 2 & 3 & 4 & 5 & 6 & $(-)$ \\
\hline & & & & & & & & \\
\hline
\end{tabular}

Figura 2. Modelo Interaccional Comportamental de Tránsito

\section{TRANS-SISTEMA TRÁNSITO Y SEGURIDAD VIAL}

El Modelo Interaccional Comportamental de Tránsito se funda a partir de la relación dialéctica, constructivista, por un lado, de los comportamientos de los actores que integran los subsistemas comportamentales del Sistema Tránsito y Seguridad Vial y, por el otro, de los elementos físicos y materiales de los diferentes ambientes (pertinente y correspondiente para cada subsistema comportamental) que dan sustento, soporte y apoyo a los actores de cada subsistema para que sus comportamientos se expresen, desarrollen y progresen en cada subsistema del STSV. Los actores intervinientes son: 1) conductor, acompañante y/o pasajero y peatón; 2) policía y/o agentes de control de tránsito y autoridades judiciales; 3) padres, pareja, amigos, instructores, maestros y profesores, medios de comunicación, TICs y asociaciones sin fines de lucro; 4) actores del poder legislativo y ejecutivo y empresarios e industriales del sector. Y los elementos reales existentes que podrían intervenir, asociados a un único y determinado subsistema comportamental, serían: vehículo, infraestructura vial, transporte, entre otros (Primer Subsistema), leyes, normativas, entre otros (Segundo Subsistema), material educativo, recursos didácticos, entre otros (Tercer Subsistema); organismos gubernamentales, creación de normas regulatorias, empresas e industrias del sector, entre otros (Cuarto Subsistema); determinando el Sistema de Elementos Físicos. Constituyéndose un Trans-Sistema Tránsito y Seguridad Vial [TSTSV] (Petit, 2015) integrado por la relación 
entre actores y elementos físicos; es decir, el Trans- Sistema Transito y Seguridad Vial (ver figura 3) se integra por dos sistemas: el sistema de actores (primeramente designado Sistema Transito y Seguridad) llamado Sistema Comportamental y el sistema de elementos físicos y materiales del entorno llamado Sistema de Elementos Físicos, ambos sistemas en asociación dialéctica dan lugar a un nuevo sistema que los subsume.

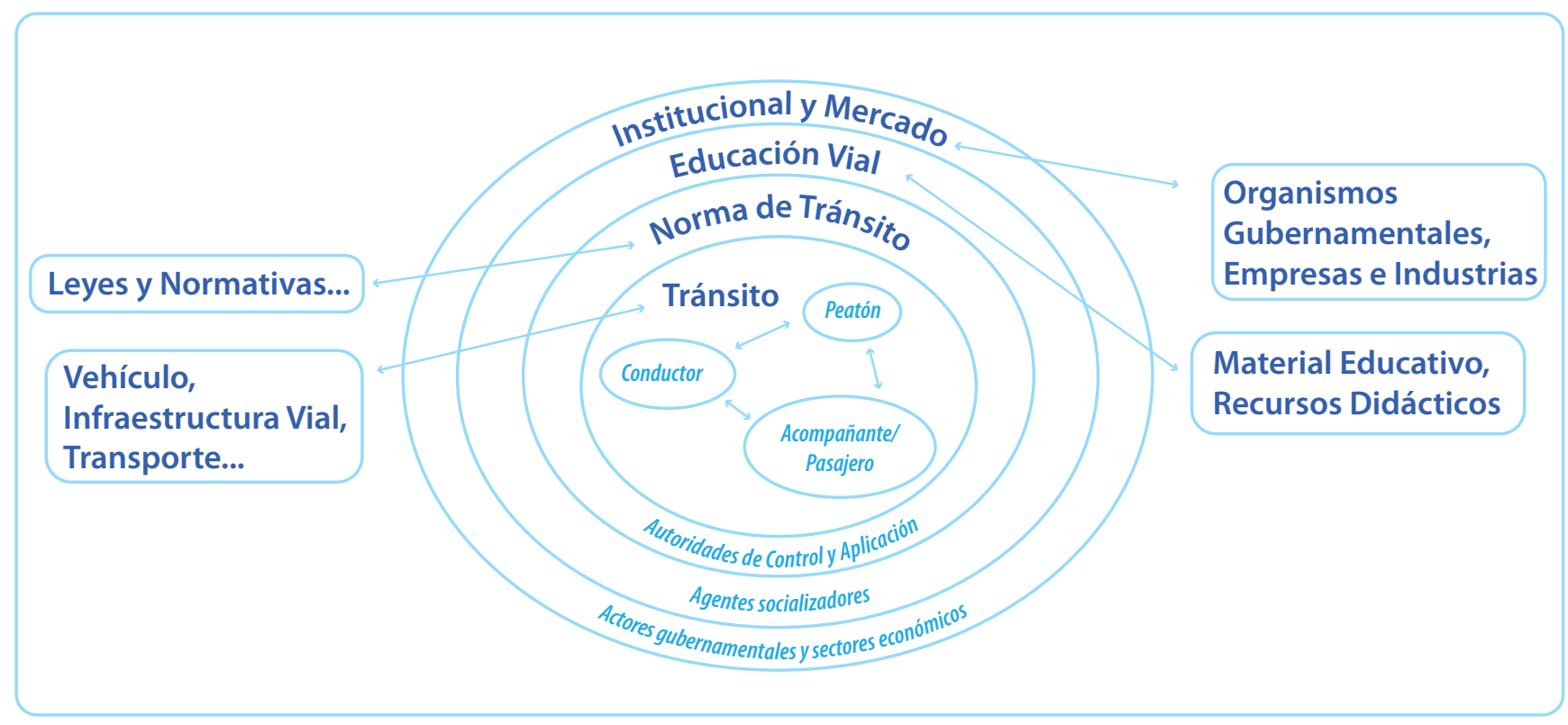

Figura 3. Trans-Sistema Tránsito y Seguridad Vial

El MICT, consolidado desde el comportamiento de los actores (factor psicosocial), posibilita al TSTSV que instaure el diálogo de las diferentes disciplinas de las Ciencias Sociales y Humanidades (Sociología, Antropología, Filosofía, Profesorados y Magisterios, Economía, Ciencia de la Educación, Ciencia de la Comunicación, Derecho, Psicología, Medicina, Trabajo Social, Ciencia Política, entre otras) con los elementos físicos del entorno (cimiento de los comportamientos y dispositivo escénico de sus acciones) cuyas disciplinas son: Ingenierías, Ciencias Exactas, Físicas y Naturales, entre otras; determinando y determinándose todos las ciencias en igual estatus.

\section{PRIMERA INSTANCIA DEL MODELO INTERACCIONAL COMPORTAMENTAL DE TRÁNSITO: DIAGNÓSTICO Y DIAGNÓSTICO EN SENDERO}

Con dicho fin se utiliza la siguiente función:

$$
\text { Interseccion }(\alpha 1, \alpha 2, \beta 1, \beta 2)
$$

Dónde:

.) $\alpha 1$ es el valor medio de la variable "A"

.) $\alpha 2$ el valor medio de la variable "B"
.) $\beta 1$ es el valor medio de la variable "YO"

.) $\beta 2$ es el valor medio de la variable "OTRO"

Por cómo se han escalado los datos:

$1 \leq \alpha 1 \leq 6$

$1 \leq \alpha 2 \leq 6$

$1 \leq \beta 1 \leq 6$

$1 \leq \beta 2 \leq 6$

Descripción:

El algoritmo Interseccion genera a partir del cálculo de pendiente y ordenada al origen la ecuación de dos rectas con los cuatro datos ofrecidos como datos de entrada y devuelve el punto de intersección de las mismas. El punto de intersección será nuestro punto sintetizador y la valoración del resultado podrá comprenderse de forma gráfica. Las fórmulas se presentan a continuación:

Cálculo de la pendiente de la recta obtenida por los datos de variable "A" y "B":

Antes de pasar a la fórmula explícita es conveniente remarcar que si bien cada variable aporta un único valor y que un punto en el plano 
de ejes cartesianos requiere dos valores (uno correspondiente al eje " $x$ " y otro al eje " $y$ ") se ha decidido tomar como coordenadas para la variable "A" el punto $(\alpha 1,0)$ y para la variable "B" el punto $(\alpha 2,-6)$ (Ver figura al final). Luego la pendiente de esta recta se obtiene como:

$$
\text { pendiente } 1=\frac{(-6-0)}{(\alpha 2-\alpha 1)}
$$

Por otro lado la ordenada al origen se obtiene como:

$$
\text { intercept } 1=0 \text {-pendiente } 1 \times \alpha 1
$$

Por último la ecuación de la recta:

\section{$f 1(\mathrm{x})=$ pendiente $1 \times x+$ intercept 1}

Análogamente se obtienen pendiente2 e intercept2 para la ecuación de la recta a partir de los datos de las variables "YO" y "OTRO". La única observación a realizar en este caso es que los puntos que se han considerado han sido $(0, \beta 1)$ para "YO" y $(6, \beta 2)$ para "OTRO".

$$
\begin{gathered}
\text { pendiente } 2=\frac{(\beta 2-\beta 1)}{(6-0)} ; \quad \text { intercept } 2=\beta 2-\text { pendiente } 2 \times 6 \\
f 2(\mathrm{x})=\text { pendiente } 2 \times x+\text { intercept } 2
\end{gathered}
$$

Por último se resuelve el sistema de ecuación que pretende hallar el punto de intersección de $f 1$ y $f 2$. Este punto tiene nuevamente dos coordenadas que llamaremos $(x i, y i)$

$$
x i=\frac{\text { intercept } 2 \text {-intercept } 1}{\text { pendiente } 1-\text { pendiente } 2} \quad y i=\text { pendiente } 1 \times x i+\text { intercept } 1
$$

Ejemplo de uso 1. El algoritmo se ha desarrollado con el software MATLAB $^{\circ}$. Para conocer los detalles, y posibilitar su utilización (replicación), podrá obtenerse el algoritmo desarrollado con el software MATLAB ${ }^{\oplus}$, más instrucciones de instalación, accediendo al vínculo:

https://drive.google.com/folderview?id=0B04gnrTGPfieYjNRYV dBRzNGUG8\&usp=sharing

Los valores del vector $\mathrm{Y}$ se obtuvieron a partir del resultado de la evaluación de dos escalas: 1) Evaluación de la frecuencia con la que los sujetos autoperciben que como conductores de autos cumplen la norma específica, dando cuenta del Comportamiento YO (Y1); obteniéndose una puntuación media de 2,3; y 2) Evaluación de la frecuencia con la que los sujetos perciben que los conductores de autos cumplen la norma específica, dando cuenta del Comportamiento OTROS (Y2); obteniéndose una puntuación media de 2,8 .
Los datos del vector $\mathrm{X}$ se obtuvieron a partir de dos mediciones: 1) Medición del estado actual del pavimento de la vía de circulación, dando cuenta del elemento físico de la Variable A; obteniéndose un puntuación de 1,9; y 2) Medición del nivel actual de señalización de la vía de circulación, dando cuenta del elemento físico de la Variable B; obteniéndose un puntuación de 0,9.

Llamado a la función, ejecución del software MATLAB ${ }^{\circledR}$

$\mathrm{X}=$ interseccion $(1.9,0.9,-2.3,-2.8)$

X: ELEMENTO FÍSICO Y ELEMENTO FÍSICO (A y B).
Y: COMPORTAMIENTO (YO, Y1, y OTROS, Y2)

Resultando del comando anterior el gráfico:

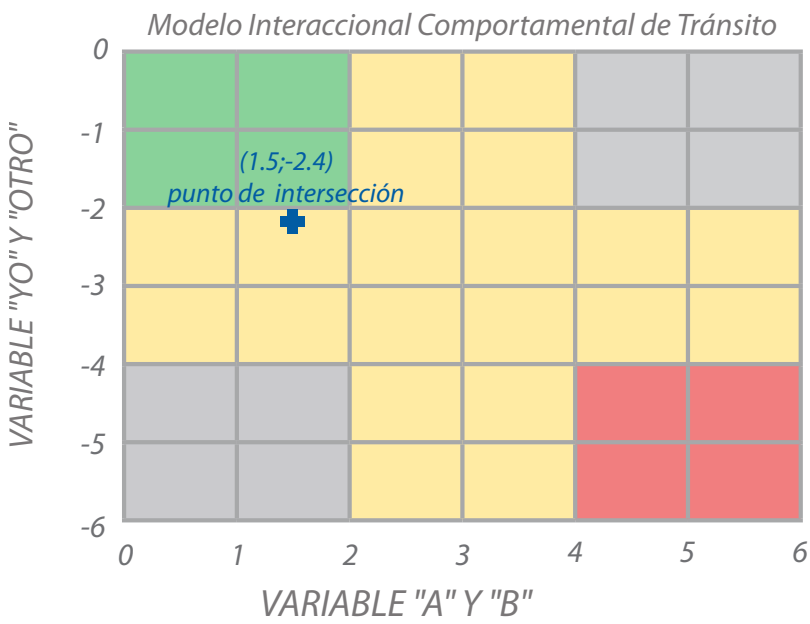

A partir del punto indicado en el plano del Modelo Interaccional Comportamental de Tránsito es posible diagnosticar la situación actual del TSTSV. Encontrándose en la condición de un Sistema en Disputa; es decir, por un lado, un adecuado estado del pavimento, más un conveniente nivel de señalización de la vía de circulación (Primer Subsistema del Sistema de Elementos Físicos), y por el otro, un modesto cumplimiento de las norma de tránsito por parte de los conductores de auto (Subsistema Tránsito del Sistema Comportamental). El diagnóstico de un Sistema en Disputa determina un sistema en una condición presente de conflicto entre el comportamiento de los actores y los elementos físicos en el que interviene los usuarios. Dando cuenta el MICT de que el favorable estado del elemento físico es insuficiente para incidir positivamente sobre el factor psicosocial. Dicho en otras palabras, lo comportamental y lo físico pertenecen a sistemas autónomos que no dan cuenta de una interacción sinérgica.

Ejemplo de uso 2. El algoritmo se ha desarrollado con el software MATLAB ${ }^{\bullet}$. Para conocer los detalles, y posibilitar su utilización (replicación), podrá obtenerse el algoritmo desarrollado con 
el software $\mathrm{MATLAB}^{\oplus}$, más instrucciones de instalación, accediendo al vínculo:

https://drive.google.com/folderview?id=0B04gnrTGPfieYjNRY VdBRzNGUG8\&usp=sharing

Los valores comportamentales de las escalas 1 y 2, vector $\mathrm{Y}$, se segmentan según el sexo (mujer y hombre), por un lado, y edad (rangos de edad: 26-30 y 18-25), por el otro. Obteniendo el Comportamiento YO (Y1), primer paréntesis en el llamado a la función, dos pares de valores: 1) Sexo mujer: $M=1,9$; y Sexo hombre: $M=3,3$ y 2) Edad rango de 26-30: $M=4,3$ y Edad rango 18-25: $M=5,7$. Y el Comportamiento OTROS (Y2), segundo paréntesis en el llamado a la función, dos pares de valores: 1) Sexo mujer: $M=2,4$ y Sexo hombre: $M=3,5$; y 2) Edad rango de 26-30: $M=4,8$ y Edad rango 18-25: $M=5,9$.

Manteniéndose inalterable el vector X.

Llamado a la función, ejecución del software MATLAB : interseccion3(1.9,0.9, [ - 1.9 -3.3 - 4.3 -5.7], [ - 2.4 - -3.5 -4.8 -5.9]) Vector Y: SEXO: MUJER (Y1), HOMBRE (Y2) - EDAD: 26-30 (Y1), 18-25 (Y2)

Resultando del comando anterior el gráfico de cuatro puntos unidos:

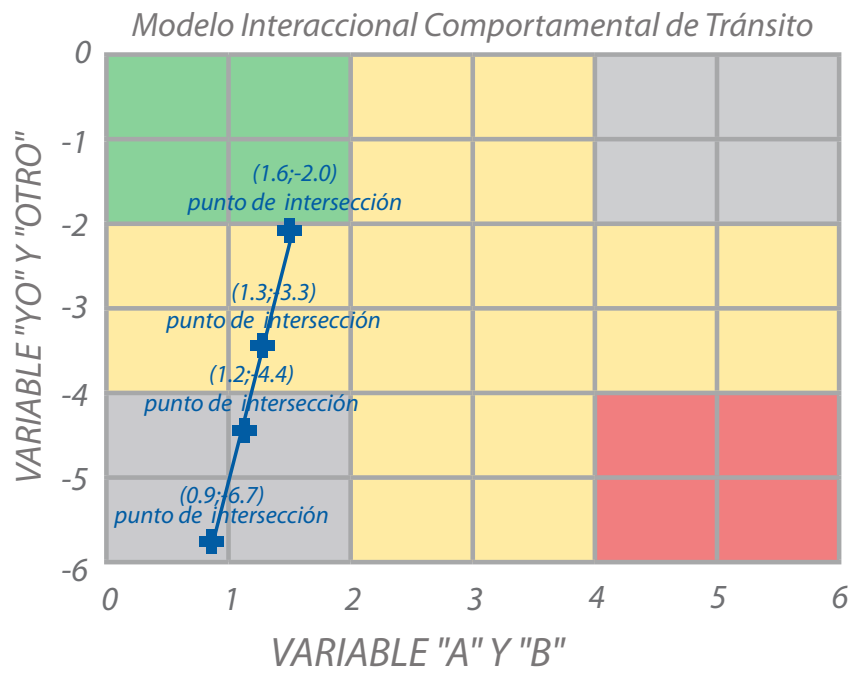

A partir de los puntos indicados en el plano es posible trazar un sendero que diagnostica la situación actual del TSTSV otorgándole movimiento a una primera evaluación estática (aquí y ahora). Expresando una doble condición y situación de diagnóstico a partir del Modelo Interaccional Comportamental de Tránsito: Sistema en Disputa y Zona de Exclusión, determinado por la pertenencia de los diferentes actores a un determinado grupo; el sendero trazado brinda Movimiento a los comportamientos.
El primer diagnóstico, ejemplo 1, no diferenciaba ninguna característica de los conductores de auto, todos integran una única categoría definida por si conduce o no conduce un vehículo. El Sendero permite diferenciar dicho comportamiento de acuerdo a su pertenecía inter- intra grupal dando cuenta de diferentes grupos de actores que presentan la misma conducta base (conductores de auto), observándose el Movimiento en relación a los diferentes subgrupos de un mismo intra grupo o de distintos inter grupos. En este ejemplo se presentan dos inter grupos: sexo y edad, ambos con dos subgrupo (intra grupos): mujer y hombre, por un lado, rango de 26 a 30 años de edad y rango de 18 a 25 años de edad, por el otro. En el primero inter grupo las mujeres presentan un Sistema en Disputa con valores menos elevados que el de los hombres, deduciéndose que las mismas realizan un mayor cumplimiento de las normas, en comparación con los hombre; y en el segundo inter grupo, ambos se encuentran en la Zona de Exclusión, situación que expresa, y da cuenta, no sólo de que el nivel de transgresión de la norma es excesivo, sino de la ausencia de relación factible entre ambos sistemas (Sistema Comportamental y Sistema de Elementos Físicos), interpretándose que la adecuada infraestructura vial de ningún modo incide en el comportamiento de los conductores de autos, repeliéndose el uno con el otro. El sistema exhibe movimientos excesivos debido a encontrarse con patrones comportamental extremos, de cierta estabilidad a inestabilidad absoluta.

\section{CONCLUSIONES}

De los dos sistemas (Sistema Comportamental y Sistema de Elementos Físicos) como unidades de análisis se deduce, por un lado, la caracterización de los procesos comportamentales de los actores y, por el otro, la descripción de los elementos físicos; ambos en relación dialógica a partir del abordaje metodológico posibilitado por el MICT, dialéctica como pretensión y determinación deliberada de conectar teoría y técnica. Las unidades de análisis resguarda el carácter molecular irreductible de sus componentes, caracterizando los patrones de una globalidad a definir. Los sistemas encuadran la complejidad de los niveles de análisis y ponderan los componentes de los subsistemas y, principalmente, sus vinculaciones. El TSTSV es la expresión de una frágil interacción entre actores y elementos físicos a partir de una estructura irreductible de los componentes de los subsistemas de los sistemas, manifestándose los diferentes modos de configuración del comportamiento en su entorno a partir de una mirada de conjunto.

La novedad no es sino la relación entre prácticas sociales y elementos físicos, la dirección que emprenda será resultante de 
la diversidad y calidad de las interacciones que se desarrollen. Es decir, la concepción dialéctica destaca la importancia de caracterizar los desarrollos y sus niveles, no funciones aisladas.

El TSTSV, como teoría, y el MICT, como técnica, plantean la heterogeneidad como factor intrínseco y constitutivo; la heterogeneidad del factor psicosocial en una multiplicidad de planos, y desde una pluralidad de perspectivas, fusionado con variedad de elementos reales. Heterogeneidad que desamarra la dicotómica: psicosocial versus físico-material, constituyéndose el MICT en un mecanismo tecnológico que operacionalizado una totalidad dinámica. Dinámica dialéctica de los conflictos y contradicciones como principio de construcción, de lo disímil a lo igual y componer una síntesis; el TSTSV como lo posible, resultante del conflicto de articulación entre lo psicosocial y los elementos reales, o dicho de otro modo: entre teoría y técnica.

La teoría del TSTSV establece que cada sistema no está predeterminado sino que dependen de las interacciones de actores y objetos, emergiendo cierta interdependencia de un sistema respecto del otro; estableciéndose sistemas complejos que establecen interdependencia, actores y objetos, y creatividad en la formación de los subsistemas, al interior de cada sistema, y en la vinculación de los sistemas (Sistema Comportamental y Sistema de Elementos Físicos). En síntesis, se mantiene la distinción entre actores y elementos físicos que dan soporte al TSTSV mientras se sostiene su dinámica de interdependencia, integridad que incluye lo disímil; un único, y abierto, observable y coordinado Trans-Sistema Tránsito y Seguridad Vial: individuos y entornos.

\section{REFERENCIAS BIBLIOGRÁFICAS}

1. Huguenin, R., \& Rumar, K. (2001). Models in Traffic Psychology. En P. E. Barjonnet (Ed.), Traffic Psychology Today (pp. 31-62). Boston: Kluwer Academic publishers.

2. Iversen, H. (2004). Risk-taking attitudes and risky driving behaviour. Transportation Research Part F: Traffic Psychology and Behaviour, 7(3), 135-150.

3. Izquierdo, J. D. D. \& Torres, R. D. (2009). Hacia una sociología de la seguridad vial: Del “factor humano" al "Factor social”. Praxis sociológica, 13, 148-172.

4. Izquierdo, J. D. D., Torres, R. D. \& Martínez, L. (2013). Control social del tráfico y empoderamiento social. Grupos de riesgo: jóvenes y mayores. Barataria. Revista Castellano-Manchega de Ciencias Sociales, 16, 161-176.

5. Ledesma, R.D., Poó, F.M. \& Montes, S.A. (2011). Psicología del tránsito: logros y desafíos de la investigación. PSIENCIA. Revista Latinoamericana de Ciencia Psicológica, 3(2), 108-119.

6. Lopez-Araujo, B. \& Osca Segovia, A. (2007). Factores explicativos de la accidentalidad en jóvenes: Un análisis de la investigación. Revista de Estudios de Jóvenes, 79, 75-89.

7. Michon, J. A. (1985). A critical review of driver behaviour models: What do we know, what should we do? En L. Evans \& R. C. Schwing (Eds.), Human behaviour and traffic safety (pp. 487-525). New York: Plenum Press.

8. Näätänen, R. \& Summala, H. (1974). A model for the role of motivational factors in drivers' decision-making. Accident Analysis E Prevention, 6, 243-261.

9. Persaud, B., Lord, D. \& Palmisano, J. (2002). Calibration and Transferability of Accident Prediction Models for Urban Intersections. Transportation Research Record: Journal of the Transportation Research Board, 1784, 57-64.

10. Petit, L. (2014). El factor humano en el Sistema Tránsito y Seguridad Vial y el Modelo Interaccional Comportamental de Tránsito. PSIENCIA. Revista Latinoamericana de Ciencia Psicológica, 6(1), 48-54.

11. Petit, L. (2015). Sistema tránsito y seguridad vial. Ciudad Autónoma de Buenos Aires: Editorial Universidad Abierta Interamericana.

12. Reason, J. (1990) Human Error. Cambridge: Cambridge University Press.

13. Rothengatter, T. (1997). Psychological aspects of road user behaviour. Applied Psychology: an International Review, 46, $223-234$.

14. Wilde, G. J. S. (1982). The theory of risk homeostasis: implications for safety and health. Risk Analysis, 2, $209-225$. 